\title{
Les retours d'expériences dans la gestion de situations critiques
}

Feedback systems and managing critical situations

\section{Anne-Lise Marchand}

\section{(2) OpenEdition}

\section{Journals}

Édition électronique

URL : http://journals.openedition.org/activites/2564

DOI : 10.4000/activites.2564

ISSN : 1765-2723

\section{Éditeur}

ARPACT - Association Recherches et Pratiques sur les ACTivités

\section{Référence électronique}

Anne-Lise Marchand, «Les retours d'expériences dans la gestion de situations critiques », Activités [En ligne], 8-2 | octobre 2011, mis en ligne le 15 octobre 2011, consulté le 19 avril 2019. URL : http:// journals.openedition.org/activites/2564; DOI : 10.4000/activites.2564

\section{(9) $\odot \Theta \Theta$}

Activités est mis à disposition selon les termes de la licence Creative Commons Attribution - Pas d'Utilisation Commerciale - Pas de Modification 4.0 International. 


\title{
Les retours d'expériences dans la gestion de situations critiques
}

\author{
Anne-Lise Marchand \\ Laboratoire d'Ergonomie, Centre de Recherche sur le Travail et de Développement, \\ CNAM- 41 rue Gay-Lussac 75005 Paris \\ annelisesibra@orange.fr
}

\begin{abstract}
Feedback systems and managing critical situations. We understand feedback as a process of collecting and sharing information about the management of critical situations affecting individuals in a complex system. Most episodes considered in studies on feedback are about individual experience (individual feedback) or are proposed by the organisation (organisational feedback). There are fewer studies on experiential narratives (collective feedback), even though such accounts from members of a group are common and are considered useful in safety management. This study aims to investigate the use of different feedback in critical situations and to test whether experiential narratives are called upon and used in critical situations. Interviews were conducted with experienced fighter pilots of the French Air Force, who were asked to give an account of how they had handled a problematic situation. The interviews were conducted in a nondirective manner so that the pilots could spontaneously mention the episode during their account. The results indicate that such episodes are often mentioned and are considered useful in dealing with critical situations. They also show a preponderance of individual feedback, then collective feedback, over organisational feedback in critical situation management.
\end{abstract}

\section{KEYWORDS}

Feedback, experiential narratives, critical situation, episodic knowledge, military aviation

\section{1.- Introduction}

Dans les systèmes complexes, certaines situations problématiques surviennent pour lesquelles il n'existe aucune procédure connue ou adaptée. Autrement dit, ces situations possèdent des caractéristiques qui font d'elles des situations uniques. Par ailleurs, leur complexité et leur rareté obligent chaque individu à réagir à ces situations à partir des ressources limitées auxquelles il a accès (Jonassen \& Hernandez-Serrano, 2002). Ces situations sont donc uniques et chacun y réagit différemment.

Cette singularisation, à la fois de chaque problème et de chaque individu qui y est confronté, rend ces situations difficiles à anticiper (Bisseret, 1984). Les dispositifs traditionnels de formation qui sont consacrés à la gestion de ces situations critiques considèrent des catégories de situations connues et permettent aux individus d'acquérir des solutions adaptées : ainsi, un pilote confronté à une panne d'alternateur en vol peut utiliser la check-list correspondante pour gérer la situation. En revanche, ces dispositifs de formation sont souvent insuffisants pour apprendre à l'individu à adapter son action lorsque ces situations s'écartent de ces catégories, par exemple lorsque le pilote est confronté à une panne d'alternateur au sol. Dès lors, il apparaît utile d'identifier et d'exploiter toutes les ressources susceptibles d'aider l'individu à gérer ces situations atypiques. Parmi ces ressources, diverses études signalent l'utilisation d'épisodes (Gick \& Holyoak, 1983; Jonassen \& Hernandez-Serrano, 2002). Les organisations confrontées aux risques peuvent proposer ces épisodes à travers un système de retour d'expérience organisationnel. Si ce dispositif permet la modification d'éléments facteurs de risque pour le système (Gauthey, 2008), on ne mesure toutefois pas aujourd'hui comment il participe à la gestion des événements imprévus in situ : autrement dit, le retour d'expérience organisationnel 
est-il utile aux individus confrontés à un risque immédiat?

Parallèlement, il est parfois tenté d'organiser les pratiques informelles et locales de retour d'expérience pour les intégrer au retour d'expérience organisationnel (Gautier, 2005). En effet, plusieurs études (Orr, 1996; Pétolas, 2005) indiquent que ces pratiques non organisées et réalisées au sein des collectifs de travail participent également à la gestion des situations imprévues à travers le partage d'épisodes. Ce retour d'expérience collectif semble présenter un intérêt pour la gestion des risques, mais le fait de le formaliser ne risque-t-il pas d'en éliminer certains bénéfices? Autrement dit, les caractéristiques du retour d'expérience collectif ne permettraient-elles pas justement la constitution de ressources complémentaires à celles proposées par le retour d'expérience organisationnel? Avant de vouloir intégrer systématiquement le retour d'expérience collectif au dispositif organisationnel, il s'agit donc d'en étudier les atouts potentiels.

L'objectif de cette étude est d'identifier et d'évaluer les éventuels bénéfices du retour d'expérience collectif pour la gestion de risques immédiats, parallèlement à ceux du retour d'expérience organisationnel. Par ailleurs, l'individu disposant également d'épisodes vécus personnellement et susceptibles d'être utilisés pour résoudre un problème (Passeron \& Revel, 2005), il apparaît nécessaire de comparer cette forme de retour d'expérience individuel aux deux formes précédentes. Cet article présente donc tout d'abord la notion d'épisode, les mécanismes par lesquels ces épisodes peuvent être utilisés pour gérer une situation critique, puis les trois origines possibles de ces épisodes, i.e. les retours d'expérience individuel, collectif et organisationnel. Enfin, les résultats de l'étude menée ayant pour objectif de comparer l'évocation d'épisode issue des différents retours d'expérience en situation critique sont présentés et discutés.

\section{2.- Retours d'expérience et gestion de situation critique}

\section{1.- Comment les épisodes participent-ils à la construction de ressources utiles à la gestion de situation critique?}

\subsection{1.- Résolution de problème et épisode}

La gestion des situations critiques en temps réel est une composante essentielle de la sécurité des systèmes. Elle est mise en œuvre localement par des activités de résolution de problème. Ces résolutions de problèmes surviennent lorsque l'opérateur est face à une situation non identifiée, pour laquelle il ne dispose d'aucune stratégie prédéfinie pour l'aider à agir. Autrement dit, il ne dispose pas de classe d'épisodes à laquelle est associée une règle permettant de résoudre le problème posé (Rasmussen, 1983). Il doit alors élaborer une nouvelle stratégie à partir de l'ensemble des ressources qui sont à sa disposition, parfois sous contraintes temporelles (Hoc, 2004) et vitales fortes (Owen, 2007). Cette gestion de l'événement doit tenir compte à la fois de ces contraintes, mais aussi des ressources cognitives et des possibilités d'action que l'individu a à sa disposition immédiate (Rasmussen, Brehmer, \& Leplat, 1990). Parmi ces ressources, l'individu dispose notamment des épisodes qu'il a mémorisés.

L'épisode est défini comme une situation expérientielle problématique «saillante » ayant donné lieu à un traitement cognitif particulier en vue de résoudre le problème posé, et se détachant de la masse des autres épisodes de l'expérience par son caractère inhabituel, dramatique, risqué, etc. (Marchand, 2009). Il peut être rapproché de l'expérience épisodique, concept développé récemment par Rogalski et Leplat (2009), et assimilé à la définition du cas donnée par Leplat (2008); il s'agit « d'un objet, un événement, une situation constituant une unité d'analyse, cette unité étant inscrite dans un contexte». Rogalski et Leplat (2009) indiquent que ces expériences épisodiques peuvent être issues à la fois de l'expérience de l'individu, mais aussi issues de l'expérience « vécue au second degré », c'est-à-dire par des pairs du même milieu professionnel. Même s'ils indiquent qu'une expérience épisodique peut devenir un paradigme utilisable comme cas d'école, ils ne précisent pas si une expérience épisodique peut être saisie et utilisée par l'organisation. La notion d'épisode proposée ici englobe tous 
les événements problématiques auxquels l'individu peut être confronté au cours de sa vie professionnelle, c'est-à-dire les expériences vécues directement, ainsi que les expériences auxquelles l'individu est confronté de façon formelle (par l'organisation) et informelle (par ses pairs).

\subsection{2.- Construction de ressources grâce au raisonnement à base de cas}

L'individu utilise ces épisodes à travers le raisonnement à base de cas (Riesbeck \& Schank, 1989; Sauvagnac, 2000). Celui-ci consiste à évoquer un cas source pour résoudre le problème posé par un cas cible. Cette stratégie se distingue de l'analogie, car si elles sont toutes deux des stratégies de rapprochement visant à comparer un fait complexe à un autre fait relativement mieux connu, la nature du rapprochement et son objectif diffèrent; l'analogie fonctionne par association, combinaison et synthèse d'éléments constitutifs de ces faits et de leurs interactions (Zouhairi, 2010). Le but est avant tout de rapprocher les faits. A contrario, le raisonnement à base de cas analyse la structure profonde des deux cas pour les comparer et résoudre le cas complexe (Passeron \& Revel, 2005). Le raisonnement à base de cas permet à l'individu de résoudre une situation problématique en amorçant sa réflexion sous un angle nouveau grâce à la comparaison des deux situations (Gick \& Holyoak, 1980 et 1983; Orr, 1996). Les informations émergeant de cette confrontation peuvent être analysées et intégrées à la représentation que l'individu a du problème posé, et lui permettre alors de comprendre ou de résoudre ce problème.

Le raisonnement à base de cas se déroule en plusieurs étapes; confronté à un problème, l'individu va :

1 - comparer le cas cible à plusieurs épisodes candidats par appariement de ses caractéristiques, ou mapping (Riesbeck \& Schank, 1989),

2 -se remémorer de façon détaillée l'épisode choisi (Kolodner, 1997; Riesbeck \& Schank, 1989),

3 -évaluer cet épisode présélectionné (Riesbeck \& Schank, 1989),

4-interpréter le cas cible au regard des enseignements tirés de l'épisode sélectionné (Kolodner, 1997),

5 -et adapter la solution appliquée lors de l'épisode sélectionné pour répondre aux exigences posées par le cas cible ou tuning (Kolodner, 1997; Riesbeck \& Schank, 1989).

Le raisonnement à base de cas est coûteux cognitivement (Sauvagnac, 2000), mais permet, en contrepartie, à chaque épisode de se présenter dans toutes ses spécificités; l'individu peut alors analyser à nouveau chaque épisode selon les nouvelles situations auxquelles il est confronté et selon les connaissances acquises dans l'intervalle. Cela signifie que, outre la résolution du problème immédiat, ces épisodes mémorisés se prêtent à d'autres utilisations (Morin, 1989) :

- l'individu peut recouper plusieurs épisodes pour identifier une propriété commune : cela lui permet éventuellement de créer une classe de situation à laquelle associer une solution (Passeron \& Revel, 2005; Sébillotte, 1993);

- il peut définir précisément un épisode en étudiant ses différentes caractéristiques afin de mieux l'exploiter (Orr, 1996; Riesbeck \& Schank, 1989) ;

- il peut comparer un épisode singulier vis-à-vis des règles générales dans le cadre desquelles l'épisode se situe. Ce type d'analyse permet de mettre en évidence des conflits de frontières entre classes de situations préétablies et de réinterroger les règles (Passeron \& Revel, 2005; Roelens, 1989) ;

- il peut acquérir des connaissances sur lui-même (Dodier, 1996; Passeron \& Revel, 2005). Pour cela, il doit se représenter les situations exposées en se substituant à ceux qui les ont vécues, ce qui lui permet d'y observer son comportement supposé et les émotions ressenties. Ce type de comportement est notamment observé dans l'aviation (O'Hare \& Wiggins, 2000). 


\subsection{3.- Savoirs épisodiques}

Les différentes possibilités évoquées ci-dessus ainsi que la confrontation à l'épisode en lui-même permettent à l'individu de développer de nouveaux savoirs. Ces savoirs dont la construction est associée à une situation problématique spécifique sont donc des savoirs épisodiques (Baerentsen, 1996). Ils constituent l'un des quatre grands pôles du modèle KEOPS (Rogalski \& Marquié, 2004) qui permet de situer différents types de savoirs par rapport à des pôles cognitifs : le pôle K regroupe les connaissances génériques et conceptuelles, le pôle E correspond aux savoirs épisodiques, le pôle O relève des outils cognitifs opératifs, et le pôle PS concerne les catégories de situations et les procédures qui y sont associées.

Lorsqu'un individu est confronté à une situation de résolution de problème, il raisonne à un niveau de contrôle cognitif où il doit élaborer une nouvelle stratégie à partir des ressources qu'il a à sa disposition pour résoudre le problème posé (Rasmussen et al., 1990). L'élaboration de cette stratégie implique une phase analytique et une phase opérative (Rasmussen, 1983) (cf. Figure 1). On propose ici que certains savoirs épisodiques soient impliqués dans la phase analytique, et d'autres dans la phase opérative.

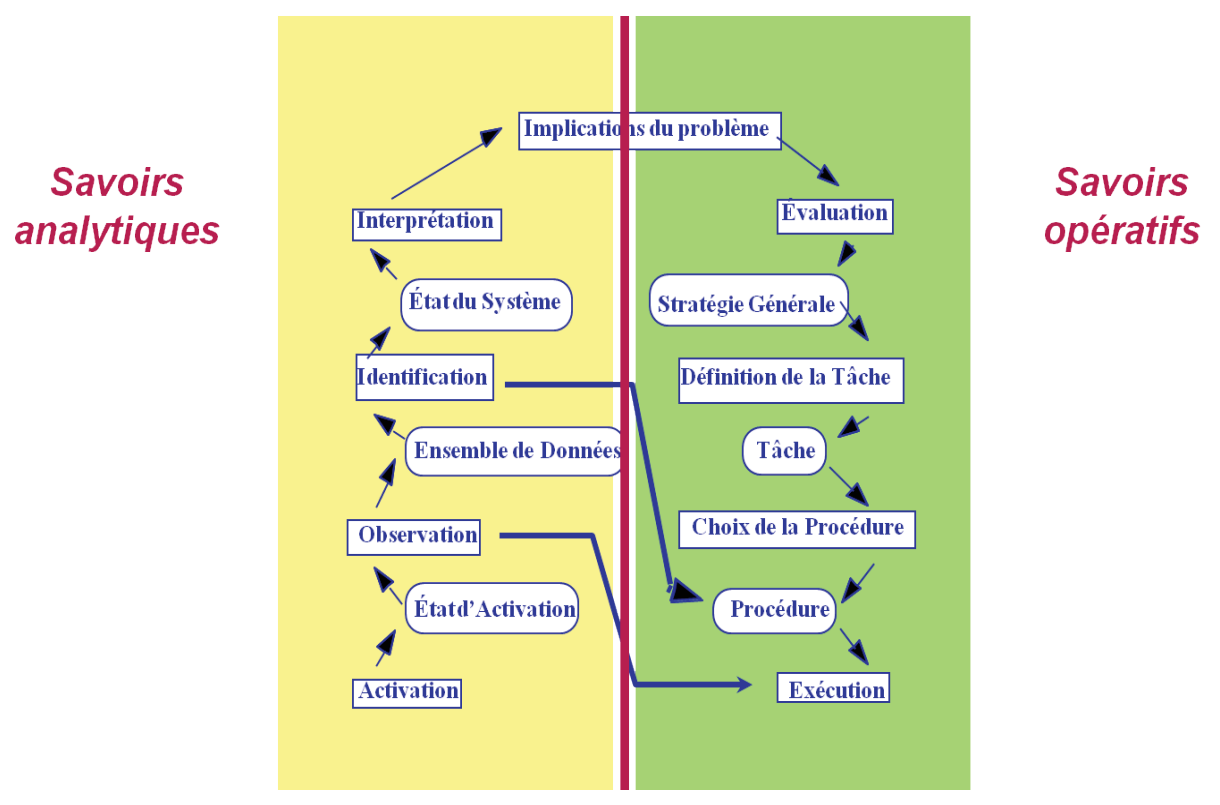

Figure 1 : Correspondance des savoirs analytiques et opératifs aux différentes phases du modèle de double échelle de Rasmussen (d'après Rasmussen, 1983)

Figure 1: The relationship between analytical and operative knowledge on the Rasmussen decision ladder model (from Rasmussen, 1983)

Les savoirs analytiques permettent de détecter une situation anormale, de la comprendre et d'établir un diagnostic. Ils concernent les connaissances d'ordre général sur le système, ses caractéristiques, la nature du ou des pièges rencontrés, leurs conséquences éventuelles et les facteurs susceptibles d'aggraver la situation. Exemple : En cas de problème de vitesse au décollage, il faut vérifier la poussée horizontale afin de discriminer si l'avion a un problème de puissance moteur ou de frein (ce savoir a été construit au cours d'un problème de frein serré au décollage).

Les savoirs opératifs concernent la mise en œuvre des stratégies de l'opérateur en réaction à la situation anormale. Ces savoirs comprennent la nature de l'action à entreprendre, l'évaluation des risques associés à la solution choisie et les conditions dans lesquelles on peut mettre en œuvre ces actions. Exemple : En Afrique, les pistes et le contrôle aérien ne sont pas toujours de bonne qualité : il vaut donc mieux parfois prendre plus de risques pour atterrir sur un terrain connu (risque météo, manque de carburant). Ce savoir a été construit lors d'un déroutement météo en Afrique. 
Les savoirs épisodiques peuvent également concerner une autre dimension de la résolution de problème; la dimension réflexive. Il s'agit de la capacité de l'individu à analyser ses propres savoirs pour les améliorer ou les appliquer à de nouvelles situations. Or, une meilleure connaissance de l'individu par lui-même peut permettre de limiter les aléas liés à ses propres connaissances et compétences dans le processus de gestion d'une situation critique (Valot, 1998). Cette dimension réflexive comprend trois applications différentes (Chartier \& Lautrey, 1992) :

- transfert d'un savoir à une situation semblable (persistance d'une procédure acquise), i.e. la prise de conscience par l'individu de sa possibilité à répéter une stratégie qui correspond à ses préférences. Par exemple, un pilote se découvre particulièrement mal à l'aise en vol de nuit,

- transfert d'un savoir à une situation analogue (mise en application dans d'autres situations), i.e. la possibilité de reproduire une stratégie donnée dans des circonstances proches, ou généralisation. Par exemple, un pilote prend conscience suite à un débriefing trop long, que tout exposé professionnel (briefing, débriefing, exposé thématique, etc.) supérieur à 30 minutes devient inefficace,

- transfert d'un savoir à des situations nouvelles, ou apprentissage issu de l'expérience. Le sujet devient capable « d'apprendre à apprendre », c'est-à-dire de reproduire un processus réflexif spécifique dans différentes situations. Par exemple, un pilote comprend que remettre en cause des données contradictoires lui permet d'éviter des erreurs (en vol, mais aussi lors de la préparation au sol ou pour la gestion de son équipement).

L'ensemble de ces savoirs épisodiques (analytiques, opératifs et réflexifs) permet à l'individu d'être mieux « outillé » face à une situation critique; ils lui permettent d'apprendre à affronter des situations non ordinaires et à risques, à la fois parce qu'ils proposent des savoirs associés à des situations atypiques (analytiques et opératifs), mais aussi parce qu'ils permettent la construction de savoirs par l'individu sur lui-même (réflexifs) (Marchand 2009). En ce sens, les savoirs épisodiques participent à la résilience de l'individu, puisqu'ils lui permettent d'adapter son fonctionnement afin d'assurer son activité dans des situations critiques imprévues (Hollnagel, 2010).

\section{2.- Coexistence de différents retours d'expériences}

\subsection{1.- Différentes origines de l'épisode}

L'épisode peut avoir trois origines différentes :

1 - il peut être issu de la confrontation directe de l'individu avec des événements imprévus, ce qui sera appelé ici retour d'expérience individuel. Cette origine des épisodes est largement développée par la littérature sur le raisonnement à base de cas (Jonassen \& Hernandez-Serrano, 2002; Riesbeck \& Schank, 1989) ou la casuistique (Passeron \& Revel, 2005); ce point ne sera donc pas développé ici ;

2-il peut être mémorisé à partir d'un récit expérientiel, c'est-à-dire de la narration d'une expérience individuelle en lien avec l'activité professionnelle survenue à l'un de membres du collectif de travail (Orr, 1996). Cette pratique est identifiée comme participant au retour d'expérience collectif (Marchand, 2009) ;

3-l'épisode peut être proposé par l'organisation par le biais de base de données (Gauthey, 2008). Ce type de pratique formalisée est appelé ici retour d'expérience organisationnel.

Le plus souvent, les épisodes considérés et étudiés par la littérature sont issus des retours d'expérience individuels (Passeron \& Revel, 2005) et organisationnels (Gaillard, 2005). Plus rares sont les études concernant les épisodes issus de pratiques locales, informelles et collectives. Il apparaît cependant que les épisodes utilisés pour la résolution de problème, notamment sous contraintes temporelles fortes, sont d'origines variables (individuelle, collective ou organisationnelle) : on peut, dès lors, s'interroger sur l'influence de ces différentes origines sur la quantité et la qualité des savoirs que les épisodes supportent. 


\subsection{2.- Le retour d'expérience collectif}

Appelé parfois « Rex au niveau de la branche professionnelle » (Gauthey 2008), le retour d'expérience collectif est constitué des pratiques locales et non organisées des opérateurs entre eux pour constituer des outils d'aide à la décision pour la gestion des risques (Gautier, 2005). Parmi ces pratiques s'échangent des récits expérientiels (Orr, 1996; Gautier, 2005) au sein du collectif de travail, soit un groupe partageant le même rapport aux objets, aux valeurs, aux relations et aux individus liés à une même activité, et utilisant le même référentiel opératif (Cuvelier \& Caroly, 2009).

Un récit expérientiel est la narration d'un épisode expérientiel remarquable d'un pair à un autre concernant leur activité. Ces récits sont le plus souvent des narrations de faits réels ou fortement inspirés de faits réels (Seely Brown, Denning, Groh, \& Prusak, 2004) qui permettent aux individus de partager leur expérience d'événements critiques (Owen, 2007). Ces récits peuvent servir à illustrer un propos ou à montrer un point spécifique de l'activité, parfois inexprimable sous forme de règle (Linde, 2001). Ils permettent aussi d'économiser la mise en pratique de certains exercices trop dangereux ou trop coûteux (Morin, 1989). Ces récits surviennent de façon spontanée, le plus souvent au cours de la formation ou lors d'échanges informels (Marchand, 2005). Dans l'exemple suivant, un instructeur pilote de chasse raconte à un élève, l'expérience qu'il a vécue concernant un problème lors d'une série de manœuvres effectuées par une patrouille de deux avions rapprochés de quelques mètres :

« Tu peux te retrouver avec des écarts de 300, 400, 500 litres entre un leader et un numéro 2, juste pour un problème d'étagement dans la tenue de la patrouille, juste à cause d'un pilotage qui n'est pas souple. C'est un truc fou. Quand j'étais jeune en escadron, j'ai fait écourter une mission COMAO parce que je ne comprenais pas bien ce que me faisait faire le leader : on était à deux avions, et vu que je tenais mal ma place (j'étais sans arrêt en train de passer de plein gaz à plein réduit) ça a donné 400 litres de différence. Ça fait deux fois le pétrole d'un epsilon. Juste sur un truc plein gaz/plein réduit. C'est énorme. Bon je ne l'ai plus jamais refait depuis, mais... c'est un truc, il faut absolument que tu fasses à l'idée que tu dois rassembler juste en coupant la trajectoire, sans rajouter de gaz. »

À l'écoute du récit, l'individu peut s'identifier aux protagonistes de l'épisode raconté et essayer de résoudre le problème exposé (Soulier \& Caussanel, 2004). Il analyse alors les raisons du succès ou de l'échec de la méthode employée pour résoudre le problème, ce qui lui évite de reproduire des stratégies en les automatisant directement (Joung, Hesketh, \& Neal, 2006). La plupart des personnels navigants interrogés sur la question attribuent à cette pratique un fort potentiel d'aide à la gestion de situation critique (Marchand, 2005).

\subsection{3.- Principes et limites du retour d'expérience organisationnel}

Le retour d'expérience organisationnel est la capitalisation, l'analyse et la distribution de l'information par l'organisation, le groupe ou le site, aux différents acteurs du système (Gauthey, 2008). Cette démarche vise à identifier et à comprendre les écarts du système, grâce à l'analyse des causes et des enchaînements de ces événements (Gautier, 2005; Gauthey, 2008). Elle se décline en trois objectifs complémentaires (Gauthey, 2008) :

- l'analyse d'événements spécifiques ou Rex événementiel,

- la mise en valeur de pratiques positives ou Rex positif,

- la détection d'événements précurseurs ou Rex à signaux faibles.

Ce dispositif doit permettre de construire des barrières de défense de façon à ce que les événements critiques identifiés ne surviennent plus (Falzon, 1998; Gaillard, 2005).

Pour répondre à ces objectifs, les organisations confrontées au risque collectent et capitalisent des épisodes issus des expériences individuelles vécus au sein de l'organisation. Ces épisodes sont saisis dans une base de données de retour d'expérience qui les trie et les indexe par thématiques. Certains problèmes d'usage apparaissent cependant (Urban, 2004; Chaskiel, 2009; Mbaye, Kouabenan, \& Samin, 2009) : interface inaccessible, relativité de la qualité des informations due à l'anonymat, 
méfiance due à la possibilité d'utiliser le retour d'expérience comme processus d'identification de responsables en cas d'accident, etc. En dépit des services rendus par ces bases de données, comme l'identification de problèmes organisationnels ou la mise en place de procédures plus adaptées pour certaines classes de situations identifiées, elles ne remplissent pas toutes les fonctions qu'on attendait d'elles (Gaillard, 2005).

Leur défaut majeur est de ne pas permettre de classifications, et donc de lectures multiples et évolutives des épisodes qu'elles contiennent (Sauvagnac, 2000) : chaque épisode est intégré à une classe de situations selon les caractéristiques prédéfinies auxquelles il répond. Les recherches dans la base de données ne peuvent se réaliser ensuite qu'à partir de ces caractéristiques ou qu'à partir de mots clefs. Cela signifie qu'un épisode ne devient visible que s'il correspond aux critères ou aux mots clefs saisis; il n'est accessible et consultable que dans cette seule perspective, alors que ses caractéristiques pourraient correspondre à une multitude d'autres systèmes de catégorisations. Ainsi, un épisode peut ne jamais être consulté en dépit de son intérêt, parce que les mots clefs ou les catégorisations saisies initialement, ou lors de la consultation ne l'incluent pas dans la recherche. Ces problèmes de catégorisations sont inhérents à tout système non évolutif, ce qui est le cas de la plupart des bases de données de retour d'expérience (Paries, Merrit, Schmidlin, \& Speyer, 1999).

\section{3.- Étude menée}

\section{1.- Problématique}

Différents types d'épisodes peuvent donc être utilisés par l'individu pour gérer une situation critique. Ils proviennent de trois retours d'expérience différents; individuel, collectif, et organisationnel. L'hypothèse testée ici est que ces trois types d'épisode peuvent être évoqués en situation critique pour aider l'individu à gérer le problème rencontré, notamment les épisodes issus du retour d'expérience collectif. Par ailleurs, les proportions dans lesquelles ces épisodes sont évoqués selon leur origine, la quantité de savoirs épisodiques qui peut leur être associée, et la qualité de l'aide apportée sont évaluées.

\section{2.- Méthodologie}

Cette hypothèse nécessite d'étudier l'évocation et l'usage d'épisodes en situation de résolution de problème. Le terrain choisi est celui de l'aviation de chasse française. Cette activité correspond à nos besoins dans la mesure où les personnels navigants (pilotes et navigateurs) doivent quotidiennement y gérer des risques vitaux sous contraintes temporelles fortes. Par ailleurs, le partage de récits expérientiels y est très courant (Marchand, 2005).

Étudier l'usage d'épisodes en situation critique comporte une difficulté méthodologique importante, puisqu'il est impossible d'accéder à la gestion cognitive des situations critiques in situ : d'une part en raison de leur caractère imprévisible, et d'autre part parce qu'il serait dangereux de demander à l'opérateur confronté à une situation critique de verbaliser à voix haute son activité cognitive simultanée; le fait de réaliser cette double tâche pourrait pénaliser l'opérateur en limitant ses ressources et lui faire courir un risque vital important.

Le recours méthodologique choisi a été d'organiser un entretien où le sujet avait pour consigne de parler librement de la gestion qu'il avait réalisée d'une situation critique rencontrée dans le passé. L'entretien était focalisé sur l'événement de façon à laisser au sujet la possibilité de mentionner spontanément l'évocation d'un épisode dans sa gestion de la situation. Si aucune mention n'était faite d'évocation d'un épisode durant le récit de la gestion de la situation critique, il était demandé explicitement à la fin de l'entretien : « avez-vous pensé à des situations similaires à ce moment-là ? ». Si le sujet répondait par l'affirmative, il lui était alors demandé une description de l'épisode évoqué. À chaque mention d'évocation (spontanée ou non) d'épisode, une série de questions était posée au 
sujet :

- la description de l'épisode évoqué et de la solution éventuellement appliquée : «pouvez-vous décrire ce souvenir précisément? »,

- l'évaluation par le sujet de l'efficacité de cette solution : «La solution mise en œuvre dans cet épisode était-elle une solution efficace ou inefficace? Pourquoi ? »,

- l'origine de l'épisode (individuelle, collective, organisationnelle) : «d'où vient ce souvenir? »,

- l'impact que le sujet attribue à cette évocation sur sa gestion du problème (positif, négatif, polluant ou neutre) : «comment qualifieriez-vous l'impact de cette évocation sur votre gestion du problème rencontré? »,

- les savoirs associés à cet épisode à travers le type d'aide éventuellement apportée par l'évocation : «cet épisode vous a-t-il aidé? Si oui, comment?».

L'ensemble des données enregistrées en audio a été transcrit. Les différents épisodes évoqués ont été identifiés comme épisode d'origine individuelle, collective ou organisationnelle. Les savoirs épisodiques associés à chaque épisode évoqué ont été catégorisés. Les savoirs sélectionnés répondaient à la définition suivante : toute information liée à l'épisode évoqué susceptible d'être pertinente dans d'autres contextes de l'activité. Par exemple, «les cumulonimbus c'est traitre » ou « quand il y a un doute, c'est qu'il n'y a pas de doute » sont des informations potentiellement utiles dans d'autres contextes, alors que « la nuit est tombée vite » ne l'est pas.

Chaque savoir a ensuite été associé à une dimension spécifique, analytique, opérative ou réflexive :

- exemple de savoir analytique : «c'est un piège classique ». Il s'agit d'une caractéristique de la situation rencontrée qui permet de mieux diagnostiquer le problème,

- exemple de savoir opératif : «on ne rassemble pas au gaz lorsqu’on vole en patrouille ». Il s'agit d'une règle d'action spécifique aux exercices en patrouille serrée, ou il faut toujours maintenir son appareil dans la même configuration que celui de son leader,

- exemple de savoir réflexif : «En général, en exercice de [tenue de patrouille], il ne faut pas craquer, parce que la situation peut vite dégénérer si on comprend mal le geste qu'a fait l'autre avion. Et pour moi, ce n'est pas gagné ». Le sujet indique qu'il sait avoir des difficultés à garder son calme lors des exercices de tenue de patrouille.

Cette méthodologie a été testée au préalable auprès de 2 pilotes de chasse et 2 navigateurs. Le recueil de données s'est ensuite déroulé sur 2 sites auprès de 33 sujets appartenant à 4 escadrons différents. Ces opérateurs étaient tous des personnels navigants militaires expérimentés (au moins trois ans d'expérience en escadron de chasse). Treize d'entre eux volaient sur avion monoplace, et 20 sur chasseur biplace (10 pilotes et 10 navigateurs).

\section{4.- Résultats}

\section{1.- Évocation des épisodes selon leur origine}

Chaque sujet interrogé a raconté une situation critique personnellement vécue. Ces situations concernaient des problèmes variés, tels qu'un bombardement en territoire hostile, un déroutement en Afrique, ou un problème de météo grave. Sur 33 sujets interrogés, 24 sujets ont évoqué au moins un épisode, et 4 sujets en ont évoqué deux, avec dans ce cas systématiquement une prédominance déclarée de l'un des épisodes sur l'autre. Aucun épisode évoqué n'était semblable à un autre; certains étaient dramatiques (collision de deux appareils avec le décès de collègues), d'autres relativement anodins et sans conséquence (oubli détecté d'un outil dans la cabine de pilotage). Ils présentaient toujours un rapport avec la situation en cours, que ce rapport soit d'ordre général (pays en guerre) ou focalisé sur un élément spécifique (le fait de connaître ou non le terrain de déroutement). 
Parmi les 24 sujets ayant évoqué au moins un épisode, 19 l'ont fait spontanément. Cinq autres sujets l'ont fait sur demande explicite. Neuf sujets ont déclaré n'avoir pas évoqué d'épisodes lors de la gestion de la situation critique racontée. Ces proportions indiquent que le recours à l'épisode est important lors de gestion de situation critique, puisque plus de 2 sujets sur 3 y ont recours. Les 28 épisodes évoqués se partagent entre (cf. Figure 2):

- 16 épisodes issus de l'expérience de l'individu,

- 11 épisodes issus du collectif,

- 1 épisode issu de l'organisation.

\section{2.- Efficacité des solutions proposées par les épisodes évoqués}

Sur 24 épisodes évoqués évalués ${ }^{1}, 22$ épisodes sont estimés avoir eu un impact positif dans la gestion de la situation critique. Un épisode est considéré comme ayant eu un impact négatif dans la gestion de la situation, et un autre épisode comme ayant eu un impact polluant.
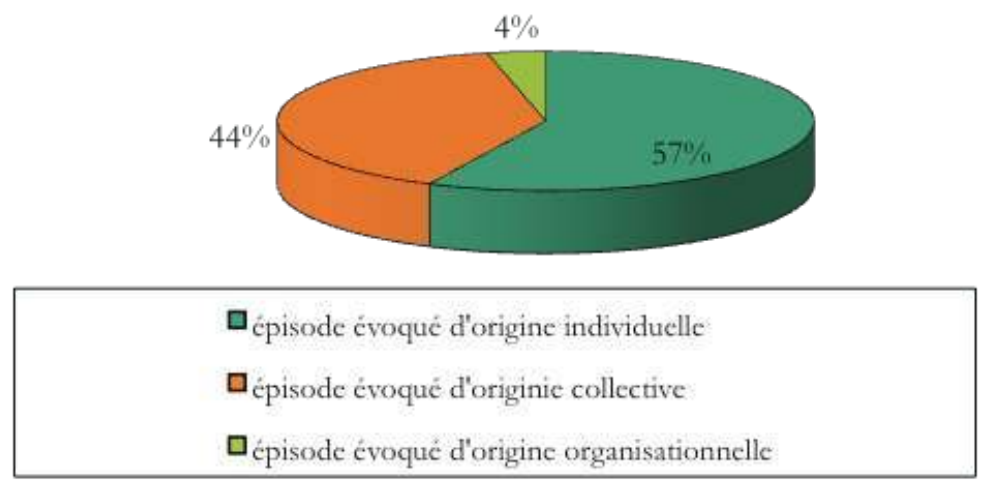

Figure 2 : Distribution des épisodes évoqués selon leur origine

Figure 2: Distribution of the episodes, by origin

Les solutions proposées pour ces 24 épisodes évoqués ont été évaluées par les sujets comme efficaces pour 14 épisodes et inefficaces pour 8 épisodes (deux des épisodes ne présentant pas de solution). Les décisions prises par les sujets dans la gestion des situations critiques auxquelles ils étaient confrontés directement ont été à $100 \%$ cohérentes avec le jugement émis sur la solution associée à l'épisode évoqué : la décision réellement prise était identique à celle prise dans l'épisode évoqué lorsque la solution était évaluée comme efficace, et inverse à celle prise dans l'épisode évoqué lorsque cette solution était évaluée comme inefficace (cf. Tableau 1). Les solutions mises en œuvre dans les 9 situations sans évocations d'épisodes n'ont pas été étudiées.

\section{3.- Proportions des différents savoirs épisodiques associés aux épisodes}

Plusieurs types d'informations et d'aides différentes ont été mentionnés par les sujets lorsqu'ils expliquaient pourquoi ils avaient évoqué un épisode en situation critique : ces différentes aides ont été catégorisées en différents savoirs. Par exemple :

- «ça m'a permis de comprendre que c'était un piège sournois, et d'y voir plus clair sur la situation » est une aide au diagnostic et correspond à un savoir analytique,

- «du coup je me suis demandé si ça ne valait pas le coup de faire comme lui avait fait, plutôt que de suivre la procédure bêtement » constitue une recherche d'alternative et correspond à un savoir opératif,

- «je me suis détendu parce que c'était déjà arrivé et que le gars qui s'en était sorti n'avait pas

1. Concernant les quatre sujets ayant évoqués deux épisodes, seul l'épisode déclaré prédominant a été pris en considération dans la mesure où le déroulement et le détail de son évocation étaient décrits plus précisément. 
beaucoup d'expérience. Il n'y avait pas de raison que je n'y arrive pas alors que j'étais plus qualifié » indique une aide à la gestion du stress et correspond à un savoir réflexif.

En moyenne, 9 savoirs différents ont été associés à chaque épisode, soit environ 3 savoirs analytiques, 2 savoirs opératifs et 4 savoirs réflexifs. Les proportions de cette distribution varient peu selon l'origine de l'épisode. En revanche, la quantité de savoirs diffère selon l'origine de l'épisode; 8 savoirs pour l'épisode d'origine organisationnelle, environ 9 savoirs par épisode d'origine collective, et plus de 11 savoirs par épisode d'origine individuelle (cf. Tableau 1).

\begin{tabular}{|c|c|c|c|c|c|c|c|c|}
\hline \multirow{2}{*}{ sujet } & \multirow{2}{*}{$\begin{array}{l}\text { épisodes } \\
\text { évoqués }\end{array}$} & \multirow{2}{*}{$\begin{array}{l}\text { origine des } \\
\text { épisodes }\end{array}$} & \multirow{2}{*}{$\begin{array}{l}\text { qualité évaluée } \\
\text { de la solution }\end{array}$} & \multirow{2}{*}{ Impact } & \multicolumn{4}{|c|}{ Savoirs } \\
\hline & & & & & analytiques & opératifs & réflexifs & total \\
\hline 1 & 1 & collectif & non efficace & positif & 3 & 3 & 6 & 12 \\
\hline \multirow{2}{*}{2} & \multirow{2}{*}{2} & collectif & efficace & positif & 4 & 3 & 4 & 11 \\
\hline & & collectif & & & 3 & 2 & 4 & 9 \\
\hline 3 & \multicolumn{8}{|c|}{0} \\
\hline 4 & \multicolumn{8}{|c|}{0} \\
\hline 5 & 1 & individuel & non efficace & positif & 3 & 2 & 6 & 11 \\
\hline 6 & 1 & individuel & efficace & positif & 2 & 0 & 3 & 5 \\
\hline 7 & 1 & collectif & non efficace & positif & 2 & 5 & 4 & 11 \\
\hline 8 & \multicolumn{8}{|c|}{0} \\
\hline 9 & \multicolumn{8}{|c|}{0} \\
\hline 10 & 1 & individuel & efficace & positif & 2 & 2 & 6 & 10 \\
\hline 11 & 1 & collectif & efficace & positif & 3 & 0 & 7 & 10 \\
\hline 12 & 1 & organisat. & non efficace & positif & 4 & 2 & 2 & 8 \\
\hline 13 & 1 & collectif & non efficace & positif & 4 & 2 & 2 & 8 \\
\hline 14 & 1 & individuel & efficace & positif & 1 & 5 & 6 & 12 \\
\hline 15 & \multicolumn{8}{|c|}{0} \\
\hline 16 & 1 & individuel & efficace & positif & 9 & 3 & 2 & 14 \\
\hline 17 & \multicolumn{8}{|c|}{0} \\
\hline 18 & 1 & individuel & non efficace & positif & 9 & 3 & 7 & 19 \\
\hline \multirow{2}{*}{19} & \multirow[b]{2}{*}{2} & individuel & efficace & polluant & 2 & 2 & 4 & 8 \\
\hline & & collectif & \multicolumn{2}{|l|}{0} & 1 & 1 & 4 & 6 \\
\hline 20 & \multicolumn{8}{|c|}{0} \\
\hline \multirow{2}{*}{21} & \multirow{2}{*}{2} & individuel & non efficace & positif & 1 & 2 & 5 & 8 \\
\hline & & individuel & \multicolumn{2}{|l|}{0} & 1 & 1 & 2 & 4 \\
\hline 22 & 1 & individuel & efficace & positif & 6 & 6 & 9 & 21 \\
\hline 23 & 1 & collectif & efficace & positif & 6 & 0 & 5 & 11 \\
\hline 24 & 1 & collectif & non efficace & positif & 3 & 2 & 0 & 5 \\
\hline 25 & 1 & individuel & efficace & positif & 6 & 10 & 8 & 24 \\
\hline 26 & \multicolumn{8}{|c|}{0} \\
\hline 27 & 1 & collectif & non efficace & positif & 2 & 1 & 3 & 6 \\
\hline 28 & 1 & individuel & efficace & positif & 2 & 2 & 3 & 7 \\
\hline \multirow{2}{*}{29} & \multirow{2}{*}{2} & collectif & efficace & positif & 4 & 3 & 4 & 11 \\
\hline & & individuel & \multicolumn{2}{|l|}{0} & 4 & 2 & 5 & 11 \\
\hline 30 & 1 & individuel & efficace & positif & 2 & 1 & 5 & 8 \\
\hline 31 & 1 & individuel & efficace & négatif & 2 & 2 & 5 & 9 \\
\hline 32 & 1 & individuel & non efficace & positif & 6 & 3 & 2 & 11 \\
\hline 33 & & & & 0 & & & & \\
\hline
\end{tabular}

Tableau 1 : Récapitulatif des données

Table 1: Data summary 


\section{5.- Différents rôles pour différents retours d'expérience}

Cette étude indique que l'évocation d'épisode en situation critique est une pratique courante et considérée comme positive dans la grande majorité des cas par ceux qui y ont recours : on peut dès lors statuer sur un rôle important, positif et utile de l'évocation d'épisode en situation critique. Les résultats obtenus permettent également d'établir dans quelle mesure l'origine des épisodes évoqués varie : les épisodes issus de l'expérience individuelle sont les plus nombreux à être évoqués, avant le retour d'expérience collectif qui fournit plus d'épisodes évoqués en situation critique que le retour d'expérience organisationnel. Par ailleurs, les épisodes les plus porteurs de savoirs sont issus tout d'abord du retour d'expérience individuel, puis collectif, puis organisationnel.

Le recours au retour d'expérience organisationnel est donc limité à la fois dans le nombre d'épisodes évoqué, mais aussi dans le nombre de savoirs épisodiques supportés. Il sert peu à la gestion de situation critique immédiate, même si les données recueillies ne permettent pas de comprendre pourquoi. Parallèlement, le retour d'expérience collectif constitue, après l'expérience personnelle, la deuxième ressource la plus sollicitée et la plus porteuse de savoirs lors de gestion de situations critiques. Dans la mesure où la pratique consistant à partager des récits expérientiels est aujourd'hui relativement méconnue et peu encouragée par les organisations, l'intérêt de cette étude réside dans le fait d'identifier cette pratique comme participant de façon importante à la gestion des risques.

Cette étude propose une distinction entre les différents retours d'expérience auxquels l'individu peut être confronté dans son activité professionnelle; individuel, collectif et organisationnel. Ceux-ci ne présentent pas les mêmes caractéristiques et donc les mêmes atouts pour la formation ou l'activité. C'est pourquoi il existe un véritable intérêt à étudier les bénéfices de chaque type de retour d'expérience : plusieurs aspects sont à identifier tels que la fiabilité du contenu des épisodes, leur potentiel pédagogique, leur souplesse d'utilisation, etc. Identifier précisément ces différentes caractéristiques permettrait d'améliorer la complémentarité des différents retours d'expérience entre eux : ainsi, le retour d'expérience organisationnel pourrait être totalement orienté vers les missions où il est efficace (détection d'événements redoutés, statistiques servant comme support d'études, etc.), et les récits expérientiels pourraient être mieux exploités au sein des collectifs confrontés à la gestion des risques. Pour cela, il serait souhaitable de valoriser les espaces de paroles informels existants, et de sensibiliser ou de former les personnels à l'intérêt du partage de récits expérientiels.

Plusieurs savoirs épisodiques ont été associés à chaque épisode; les plus nombreux à avoir été verbalisés sont les savoirs épisodiques réflexifs, ce qui confirme l'intérêt pour sa résilience, d'une bonne connaissance de l'individu par lui-même en situation critique. Toutefois, si ce concept de savoir réflexif, ainsi que d'autres concepts développés ici (épisode, savoirs analytique ou opératif) permettent de cadrer cette étude, il semble nécessaire de les éprouver à nouveau afin d'en définir plus précisément les caractéristiques et l'intérêt.

De même, la méthodologie utilisée a permis d'accéder à des informations par le biais de verbalisations spontanées a posteriori, le contexte professionnel rendant difficile tout relevé d'observations in situ. Toutefois, cette méthodologie présente la limite de ne se baser que sur des déclarations, et il serait souhaitable de renforcer la robustesse des résultats par des données objectives.

\section{RÉFÉRENCES}

Baerentsen, K.B. (1996). Episodic Knowledge in System Control. In B. Holmquist, P. Andersen, \& H. Klein (Eds.), Signs of Work : Semiosis and Information Processing in Organisations (pp. 283-323). Berlin: Walter De Gruyter.

Bisseret, A. (1984). Expert-computer aided decision in supervisory control. Paper presented at IFAC'84 Congress, Budapest. 
Chartier, D., \& Lautrey, J. (1992). Peut-on apprendre à connaître et à contrôler son propre fonctionnement cognitif? L'orientation scolaire et professionnelle, (21), 27-46.

Chaskiel, P. (2009). Les relations professionnelles de la sécurité industrielle : le REX comme outil de médiation? Numéro 2009-10 des Cahiers de la Sécurité Industrielle, Institut pour une Culture de Sécurité Industrielle, Toulouse, France (ISSN 2100-3874).

Cuvelier, L., \& Caroly, S. (2009). Appropriation d'une stratégie opératoire : un enjeu du collectif de travail, Activités, 6(2), 57-74.

Dodier, N. (1996). Ce que provoquent les infractions : Étude sur le statut pragmatique des règles de sécurité. In J. Girin \& M. Grosjean (Eds.), La transgression des règles au travail (pp. 11-37).Paris: L'Harmattan.

Falzon P. (1998). La construction des connaissances en ergonomie : éléments d'épistémologie. In M.F. Dessaigne \& I. Gaillard (Eds), Des évolutions en ergonomie (pp. 211-224). Toulouse: Octarès.

Gaillard, I. (2005). Facteurs socio-culturels de réussite du REX industriel par l'analyse bibliographique. Numéro 2008-01 des Cahiers de la Sécurité Industrielle, Institut pour une Culture de Sécurité Industrielle, Toulouse, France (ISSN 2100-3874).

Gautier, A. (2005). Retour d'expérience organisationnel et conceptualisation de l'action dans une perspective d'apprentissage pour les organisations. Information Sciences for Decision Making, 40, 704.

Gauthey, O. (2008). Le retour d'expérience : état des pratiques industrielles. Numéro 2008-02 des Cahiers de la Sécurité Industrielle, Institut pour une Culture de Sécurité Industrielle, Toulouse, France (ISSN 21003874).

Gick, M.L., \& Holyoak, K.J. (1980). Analogical problem solving. Cognitive psychology, 12, 306-355.

Gick, M.L., \& Holyoak, K.J. (1983). Schema induction and analogical transfer. Cognitive psychology, 15, $1-38$.

Hoc, J. M. (2004). La gestion des situations dynamiques. In P. Falzon (Ed), Ergonomie (pp 517-530). Paris: PUF.

Hollnagel, E. (2010). Prologue: The scope of resilience engineering. In E. Hollnagel, J. Pariès, D. Woods, \& J. Wreathall (Eds.), Resilience Engineering in Practice: A Guidebook. Ashgate: Studies in Resilience Engineering.

Jonassen, D.H., \& Hernandez-Serrano, J. (2002). Case-based reasoning and instructional design : Using stories to support problem solving. Educational Technology: Research and Development, 50(2), 65-77.

Joung W., Hesketh B., \& Neal A. (2006). Using "War Stories" to Train for Adaptive Performance: Is it Better to Learn from Error or Success? Applied psychology, 55(2), 282-302.

Kolodner, J. (1997). Educational implications of analogy : a view from case-based reasoning. American psychologist 52(1), 57-66.

Leplat, J. (2008). De l'étude de cas à l'analyse de l'activité. In : Repères pour l'analyse de l'activité en ergonomie (pp. 181-226). Paris: PUF. (Antérieurement publié dans Pistes, 2002 ; http://www.pistes.uqam. ca/v4n2/articles/van2a8.htm.)

Linde, C. (2001). Narrative and social tacit knowledge. Journal of Knowledge Management 5 (2), 160-170.

Marchand, A.L. (2005). Anecdote réflexive : une transmission de savoirs basés sur l'expérience. Mémoire de Master recherche en Ergonomie, CNAM, Paris.

Marchand, A.-L. (2009). Usage des récits expérientiels et des savoirs épisodiques dans l'apprentissage de la gestion des situations critiques. Thèse en ergonomie, CNAM, Paris.

Mbaye, S., Kouabenan, R., \& Samin, P. (2009). L'explication nä̈ve et la perception des risques comme des voies pour améliorer les pratiques de REX : des études dans l'industrie chimique et l'industrie nucléaire. Numéro 2009-08 des Cahiers de la Sécurité Industrielle, Institut pour une Culture de Sécurité Industrielle, Toulouse, France (ISSN 2100-3874). 
Morin, J. M. (1989). Histoires de vie dans un processus de formation militaire. In G. Pineau \& G. Jobert (Eds), Histoires de vies (18). Paris: L'Harmattan.

O'Hare, D. \& Wiggins, M. (2000). Remembrance of Cases Past: remembers what, when confronting critical flight events? Aviation Safety, 4(2), 277-287.

Orr, J. E. (1996). Talking about Machines. New York: Cornell University Press.

Owen, C. A. (2007). Analyse de l'activité de travail dans la gestion des situations d'urgence. @ctivités, 4(1), 207-216.

Paries, J., Merrit, A., Schmidlin, M., \& Speyer, J.J. (1999). Development of a Methodology for Operational Incident Reporting and Analysis Systems. Appel d'offres DGAC N 96/01, Lot 07.

Passeron, J.C., \& Revel, J. (2005). Penser par cas : Raisonner à partir de singularités. In J.C. Passeron \& J. Revel (Eds), Penser par cas (pp. 9-44). Paris: École des Hautes Études en Sciences Sociales.

Pétolas, M.-C. (2005). Rôle du retour d'expérience dans le développement des compétences et de la sécurité en environnement dynamique. Thèse en ergonomie, Paris VIII, Paris.

Rasmussen, J. (1983), Skills, Rules, and Knowledge: Signals, Signs and Symbols, and Other Distinctions, in Human Performance Models. IEE Transactions in systems, Man and Cybernetics, Vol. SMC-13(3), $257-$ 266.

Rasmussen, J., Brehmer, B., \& Leplat, J. (1990). Distributed Decision Making: Cognitive Models for Cooperative Work. Chichester: Wiley.

Riesbeck, C. K., \& Schank, R. C. (1989). Inside case-based reasoning. Hillsdale, New Jersey: Lawrence Erlbaum Associates.

Roelens, N. (1989). Expérience et apprentissage : faire de nécessité vertu. Éducation permanente, (100-101), 67-78.

Rogalski, J., \& Leplat, J. 2009, Des expériences dans l'Expérience. Actes du 1er colloque de didactique professionnelle, Dijon, France, 2-4 décembre 2009.

Rogalski, J., \& Marquié, J.-C. (2004). Évolution des compétences et performances. In J.-M. Hoc \& F. Darses (Ed), Psychologie ergonomique, tendances actuelles (pp 141-173). Paris: PUF.

Sauvagnac, C. (2000). La construction de connaissances par l'utilisation et la conception de procédures Contribution au cadre théorique des activités métafonctionnelles. Thèse en ergonomie, CNAM, Paris.

Sébillotte, S. (1993). Schémas d'actions acquis par l'expérience dans les représentations mentales des opérateurs : leurs utilisations et la construction de nouveaux schémas. In A. Weill-Fassina, P. Rabardel, \& D. Dubois (Eds), Représentations pour l'action (pp. 57-77).Toulouse: Octarès.

Seely Brown, J. Denning, D. Groh K. \&Prusak L. (2004). How we got into storytelling. In J. Seely Brown, D. Denning, K. Groh, \& L. Prusak (Eds), Storytelling in Organizations (pp 1-11). UK: Butterworth Heinemann.

Soulier, E., \& Caussanel, J. (2004). Apprentissage assisté par storytelling : une pédagogie de l'erreur. archive-eductice.ccsd.cnrs.fr.

Valot, C. (1998). Métacognition \& connaissances métacognitives intérêt pour l'ergonomie. Thèse en Ergonomie, Université Toulouse Le Mirail, Toulouse.

Urban, S. (2004). Les voies de progrès du retour d'expérience dans l'armée de l'air : Base facteurs humains pour la conception des systèmes homme-machine en aéronautique. Mémoire de Diplôme Universitaire en Facteurs Humains, Paris V, Paris.

Zouhairi, A. (2010). L’analogie, moteur de la pensée,.Sciences Humaines, nº 215.

\section{RÉsumÉ}

Le retour d'expérience est un processus de collecte et de partage d'épisodes de gestion de situations critiques survenues aux individus d'un système complexe. La plupart des épisodes considérés dans les études concernant 
le retour d'expérience proviennent de l'expérience individuelle (retour d'expérience individuel) ou sont proposés à l'individu par l'organisation (retour d'expérience organisationnel). Plus rares sont les études concernant les récits expérientiels (retour d'expérience collectif). Or, ces récits d'expérience issus des membres du collectif sont courants et considérés comme utiles dans la gestion des risques par les opérateurs. Cette étude vise à étudier l'usage des différents retours d'expérience en situation critique immédiate, et à tester le fait que des récits expérientiels sont évoqués et utilisés dans les situations critiques. Des entretiens ont été réalisés avec des pilotes d'avion de chasse expérimentés de l'Armée de l'Air Française. Ceux-ci devaient raconter leur gestion personnelle d'une situation problématique. Ces entretiens étaient menés d'une manière non directive de façon à ce que les sujets puissent mentionner spontanément l'évocation de l'épisode pendant leur gestion de cette situation critique. Les résultats indiquent que les évocations d'épisodes sont courantes et considérées comme utiles pour traiter une situation problématique. Ces résultats indiquent également la prépondérance du retour d'expérience individuel puis du retour d'expérience collectif en gestion de situation critique immédiate sur le retour d'expérience organisationnel.

\section{Mots CLEFS}

Retour d'expérience, récits expérientiels, situation critique, savoirs épisodiques, aviation militaire.

\section{RÉFÉRENCEMENT}

Marchand, A.-L. (2011). Les retours d'expériences dans la gestion de situations critiques. Activités, 8(2), pp. 100-113, http://www.activites.org/v8n2/v8n2.pdf

Article soumis le 26 mai 2010, accepté pour publication le 27 juillet 2011 